\title{
STABILITY AND GROWTH ESTIMATES FOR VOLTERRA INTEGRODIFFERENTIAL EQUATIONS IN HILBERT SPACE
}

\section{BY FREDERICK BLOOM}

Communicated by James H. Bramble, February 20, 1976

Let $H, H_{+}$be real Hilbert spaces with $H_{+}$dense in $H$ and $H_{+} \subset H$, algebraically and topologically; the inner products on $H, H_{+}$are denoted by $\langle$,$\rangle and$ $\langle,\rangle_{+}$, respectively. As in [1], let $H_{-}$denote the dual of $H_{+}$via the inner product of $H$ so that $H_{-}$is the completion of $H$ under the norm

$$
\|\mathbf{w}\|_{-}=\sup _{\boldsymbol{v} \in H_{+}} \frac{|\langle v, w\rangle|}{\|v\|_{+}} .
$$

By $L\left(H_{+}, H_{-}\right)$we denote the space of bounded linear operators from $H_{+}$to $H_{-}$. For $0 \leqslant t<T, T>0$ an arbitrary real number, we consider the initial-value problem

$$
\begin{gathered}
\mathbf{u}_{t t}-\mathbf{N u}+\int_{-\infty}^{t} \mathbf{K}(t-\tau) \mathbf{u}(\tau) d \tau=\mathbf{0}, \\
\mathbf{u}(0)=\mathbf{f}, \quad \mathbf{u}_{t}(0)=\mathbf{g},
\end{gathered}
$$

where $\mathbf{N} \in L\left(H_{+}, H_{-}\right)$is symmetric and $\mathbf{K}(t), \mathbf{K}_{t}(t) \in L^{2}\left((-\infty, \infty) ; L\left(H_{+}, H_{-}\right)\right)$. We also assume that

$$
\mathbf{u}(\tau)=\mathbf{U}(\tau), \quad-\infty<\tau<0,
$$

where $\mathbf{U}(t) \in C^{1}\left((-\infty, 0) ; H_{+}\right)$is prescribed and satisfies $\lim _{t \rightarrow 0^{-}} \mathbf{U}(t)=\mathbf{f}$, $\lim _{t \rightarrow 0^{-}} \mathbf{U}_{t}(t)=\mathrm{g}, \lim _{t \rightarrow-\infty}\|\mathrm{U}(t)\|_{+}=0$ and $\int_{-\infty}^{0}\|U(t)\|_{+} d t<\infty$.

In [2] we have proved the following basic result concerning solutions $\mathbf{u} \in$ $C^{2}\left([0, T) ; H_{+}\right)$for which $\mathbf{u}_{t} \in C^{1}\left([0, T) ; H_{+}\right)$and $\mathbf{u}_{t t} \in C\left([0, T) ; H_{-}\right)$. Let

$$
N=\left\{\mathrm{w} \in C^{2}\left([0, T) ; H_{+}\right) \mid \sup _{[0, T)}\|\mathrm{w}(t)\|_{+} \leqslant N^{2}\right\}
$$

for some real number $N$. Then we have

THEOREM (BLOOM [2]). Let $\mathbf{u} \in N$ be any solution of (1)-(3) and define

$$
F\left(t ; \beta, t_{0}\right)=\|\mathbf{u}(t)\|^{2}+\beta\left(t+t_{0}\right)^{2}, \quad 0 \leqslant t<T,
$$

AMS (MOS) subject classifications (1970). Primary 47G05; Secondary 45K05, 45N05.

Key words and phrases. Intergrodifferential equation, logarithmic convexity, stability, growth estimates. 
where $\beta, t_{0}$ are arbitrary nonnegative real numbers. Then provided $\mathbf{K}(t)$ satisfies

$$
-\langle v, \mathbf{K}(0) v\rangle \geqslant \kappa\|v\|_{+}^{2}, \forall v \in H_{+}
$$

with

$$
\kappa \geqslant T \gamma \sup _{[0, \infty)}\left\|\mathrm{K}_{t}(t)\right\|,{ }^{1}
$$

$F\left(t ; \beta, t_{0}\right)$ satisfies

$$
F F^{\prime \prime}-F^{\prime 2} \geqslant-2 F(2 G(0)+\beta), \quad 0 \leqslant t<T,
$$

where

$$
G(t)=E(t)+k_{1} \sup _{[0, \infty)}\|\mathbf{K}(t)\|+k_{2} \sup _{[0, \infty)}\|\mathbf{K}(t)\|
$$

with $E(t) \equiv 1 / 2\left\langle\mathbf{u}_{t}(t), \mathbf{u}_{t}(t)\right\rangle-1 / 2\langle\mathbf{u}(t), \mathbf{N u}(t)\rangle$, the total energy and

$$
\begin{aligned}
& k_{1}=\frac{1}{2} \gamma\left[T N^{4}+\left(N^{2}+2\|\mathbf{f}\|_{+}\right) \int_{-\infty}^{0}\|\mathrm{U}(\tau)\|_{+} d \tau\right], \\
& k_{2}=T N^{2} \gamma \int_{-\infty}^{0}\|\mathrm{U}(\tau)\|_{+} d \tau .
\end{aligned}
$$

The proof of this theorem proceeds via a logarithmic convexity argument due to Knops and Payne [2]. As no definiteness conditions are imposed on $\mathbf{N}$, the problem (1)-(3) is, in general, non-well-posed and the existence and uniqueness results of Dafermos [1] do not apply.

From (6) there follows a variety of results concerning the stability and growth behavior of solutions $\mathbf{u} \in N$ to (1)-(3); proofs of the sample results given below, as well as several others, along with applications to initial-boundary value problems arising in the theory of isothermal linear viscoelasticity may be found in [2] and [4]; in a forthcoming work [5] our results will be applied so as to study the stability and growth behavior of electric displacement fields in nonconducting material dielectrics.

TheOREM I (BLOOM [2]). Let $\mathbf{u} \in \mathrm{N}$ be any solution of (1)-(3) for which $E(0) \leqslant-k$, for some $k>0$. If $\mathbf{K}(t)$ satisfies $(5 \mathrm{a}),(5 \mathrm{~b})$ and

$$
\sup _{[0, \infty)}\|\mathbf{K}(t)\| \leqslant \gamma k T /\left(k_{1} T \gamma+k_{2}\right)
$$

then

$$
\|\mathbf{u}(t)\|^{2} \geqslant\|\mathbf{f}\|^{2} \exp \left\{\langle 2 f, g\rangle t /\left\|\mathbf{f}^{2}\right\|\right\}, \quad 0 \leqslant t<T,
$$

whenever $\langle\mathbf{f}, \mathbf{g}\rangle \geqslant 0$ with $\mathbf{f} \neq \mathbf{0}$.

${ }^{1} \gamma$ is the embedding constant, i.e., as $H_{+} \subset H$ topologically, $\|v\|<\gamma\|v\|_{+}, \forall v \in H_{+}$. 
Theorem II (BLoom [2]). Let $\mathbf{u} \in N$ be any solution of (1)-(3) and suppose that

$$
E(0)>-\left(k_{1} T \gamma+k_{2}\right) \sup _{[0, \infty)}\left\|\mathbf{K}_{t}(t)\right\|
$$

and

$$
\langle\mathbf{f}, \mathbf{g}\rangle 2 \sqrt{2+\epsilon}(G(0))^{1 / 2}\|\mathbf{f}\|
$$

with $\epsilon=1 / 4 \mu\langle\mathbf{f}, \mathbf{g}\rangle$, for some $\mu>0$. Then provided $\mathbf{K}(t)$ satisfies (5a), (5b), $\mathbf{u}$ satisfies

$$
\|\mathbf{u}(t)\|^{2}+\mu^{-1} \geqslant\left(\|\mathbf{f}\|^{2}+\mu^{-1}\right)\left(t / \hat{t}_{0}+1\right)^{\epsilon} \exp \left\{\delta_{\mu}\left(\hat{t}_{0} ; \epsilon\right) t\right\}
$$

for $0 \leqslant t<T$, where $\hat{t}_{0} \equiv\langle\mathbf{f}, \mathbf{g}\rangle / 4 G(0)$ and

$$
\delta_{\mu}\left(\hat{t}_{0} ; \epsilon\right) \equiv 2\left\{\frac{\langle\mathbf{f}, \mathbf{g}\rangle+\hat{t}_{0} / \mu}{\|\mathbf{f}\|^{2}+1 / \mu}\right\}-\frac{(2+\epsilon)}{\hat{t}_{0}} .
$$

THEOREM III (BLOOM [4]). Let $\mathrm{u} \in N$ be any solution of (1)-(3) where $\mathbf{K}(t)$ satisfies $(5 \mathrm{a})$ and $(5 \mathrm{~b})$. If

$$
E(0) \leqslant-k \sup _{[0, \infty)}\|\mathbf{K}(t)\|
$$

with $k \geqslant k_{1}+\gamma k_{2} / T$, then $\mathbf{u}$ satisfies

$$
\|\mathrm{u}(t)\|^{2} \leqslant A\left[\max \left(\|\mathbf{f}\|^{2},\|\mathrm{~g}\|^{2}\right)\right]^{2(1-\delta)}
$$

for $0 \leqslant t<T$, where $A>0$ and $\delta=t / T$.

THEOREM IV (BLOOM [4]). Let $\mathrm{u} \in N$ be any solution on (1)-(3) and suppose that $E(0)>-\tilde{k}$, for some $\tilde{k} \geqslant 0$. If $\mathbf{K}(t)$ satisfies $(5 \mathrm{a}),(5 \mathrm{~b})$ and

$$
\begin{aligned}
& \text { (i) } \sup _{[0, \infty)}\left\|\mathrm{K}_{t}(t)\right\|>\tilde{k} /\left(k_{1} T \gamma+k_{2}\right), \\
& \text { (ii) } \lim _{T \rightarrow \infty} \frac{1}{T} \ln \left\{\|\mathbf{u}(T)\|^{2}+\beta\left(T+t_{0}\right)^{2}\right\}=0
\end{aligned}
$$

for $\beta, t_{0}$ nonnegative constants satisfying $\beta t_{0}^{2} \leqslant\|\mathbf{f}\|^{2}$, then $\mathbf{u}$ satisfies

$$
\|\mathbf{u}(t)\|^{2} \leqslant \Phi\left(t_{0}, T ; \epsilon\right)\|\mathbf{f}\|^{2}, \quad 0 \leqslant t<T,
$$

where $\Phi\left(t_{0}, T ; \epsilon\right) \equiv 2\left(T / t_{0}+1\right)^{2+\epsilon}$ with $\epsilon=G(0) / \beta$.

\section{BIBLIOGRAPHY}

1. C. M. Dafermos, An abstract Volterra equation with applications to linear viscoelasticity, J. Differential Equations 7 (1970), 554-569. MR 41 \#4305. 
2. F. Bloom, Growth estimates for solutions to initial-boundary value problems in viscoelasticity, J. Math. Anal. Appl. (to appear).

3. R. J. Knops and L. E. Payne, Growth estimates for solutions of evolutionary equations in Hilbert space with applications in elastodynamics, Arch. Rational Mech. Anal. 41 (1971), 363-398. MR 48 \#9068.

4. F. Bloom, On stability in linear viscoelasticity, Mechanics Research Communications 3 (1976), 143-150.

5. - Stability of electric displacement fields in non-conducting material dieelectrics, (in preparation).

DEPARTMENT OF MATHEMATICS AND COMPUTER SCIENCE, UNIVERSITY OF SOUTH CAROLINA, COLUMBIA, SOUTH CAROLINA 29208 